\title{
Novel manifestations of Warburg micro syndrome type 1 caused by a new splicing variant of RAB3GAP1: a case report
}

Raziyeh Khalesi', Ehsan Razmara', Golareh Asgaritarghi ${ }^{2}$, Ali Reza Tavasoli ${ }^{3}$, Yasser Riazalhosseini ${ }^{4,5}$, Daniel Auld ${ }^{4,5^{*}}$ and Masoud Garshasbi ${ }^{1 *}$

\begin{abstract}
Background: The present study aimed to determine the underlying genetic factors causing the possible Warburg micro syndrome (WARBM) phenotype in two Iranian patients.

Case presentation: A 5-year-old female and a 4.5-year-old male were referred due to microcephaly, global developmental delay, and dysmorphic features. After doing neuroimaging and clinical examinations, due to the heterogeneity of neurodevelopmental disorders, we subjected 7 family members to whole-exome sequencing. Three candidate variants were confirmed by Sanger sequencing and allele frequency of each variant was also determined in 300 healthy ethnically matched people using the tetra-primer amplification refractory mutation system-PCR and PCR-restriction fragment length polymorphism. To show the splicing effects, reverse transcriptionPCR (RT-PCR) and RT-qPCR were performed, followed by Sanger sequencing. A novel homozygous variant-NM_ 012233.2: c.151-5 T > G; p.(Gly51llefsTer15) —in the RAB3GAP1 gene was identified as the most likely disease-causing variant. RT-PCR/RT-qPCR showed that this variant can activate a cryptic site of splicing in intron 3, changing the splicing and gene expression processes. We also identified some novel manifestations in association with WARBM type 1 to touch upon abnormal philtrum, prominent antitragus, downturned corners of the mouth, malaligned teeth, scrotal hypoplasia, low anterior hairline, hypertrichosis of upper back, spastic diplegia to quadriplegia, and cerebral white matter signal changes.
\end{abstract}

Conclusions: Due to the common phenotypes between WARBMs and Martsolf syndrome (MIM: 212720), we suggest using the "RABopathies" term that can in turn cover a broad range of manifestations. This study can per se increase the genotype-phenotype spectrum of WARBM type 1.

Keywords: PCR-RFLP, RAB3GAP1, Tetra-primer ARMS-PCR, WARBM type 1, Whole-exome sequencing

\footnotetext{
* Correspondence: daniel.auld@mcgill.ca; masoud.garshasbi@modares.ac.ir

${ }^{4}$ McGill Genome Centre, Montréal, Québec, Canada

'Department of Medical Genetics, Faculty of Medical Sciences, Tarbiat Modares University, Tehran, Iran

Full list of author information is available at the end of the article
}

(c) The Author(s). 2021 Open Access This article is licensed under a Creative Commons Attribution 4.0 International License, which permits use, sharing, adaptation, distribution and reproduction in any medium or format, as long as you give appropriate credit to the original author(s) and the source, provide a link to the Creative Commons licence, and indicate if changes were made. The images or other third party material in this article are included in the article's Creative Commons licence, unless indicated otherwise in a credit line to the material. If material is not included in the article's Creative Commons licence and your intended use is not permitted by statutory regulation or exceeds the permitted use, you will need to obtain permission directly from the copyright holder. To view a copy of this licence, visit http://creativecommons.org/licenses/by/4.0/ The Creative Commons Public Domain Dedication waiver (http://creativecommons.org/publicdomain/zero/1.0/) applies to the data made available in this article, unless otherwise stated in a credit line to the data. 


\section{Background}

Warburg Micro syndrome (WARBM) - also known as Micro Syndrome-is a rare autosomal recessive disorder with unknown true incidence. This syndrome is characterized by neurodevelopmental abnormalities such as congenital or postnatal microcephaly, severe intellectual disability, pachy- or polymicrogyria, and hypoplasia/ agenesis of the corpus callosum in addition to the ocular manifestations including congenital cataract, microcornea, microphthalmia, and optic atrophy [1, 2]. This syndrome embraces four subtypes as in WARBM type 1 (MIM: 600118) which is caused by biallelic mutations of the RAB3 GTPase-activating protein 1 gene (RAB3GAP1; MIM: 602536) [3], WARBM type 2 (MIM: 614225) by mutations of RAB3GAP2 (MIM: 609275) [4], WARBM type 3 (MIM: 614222) by mutations of the Ras-related protein Rab-18 (RAB18; MIM: 602207) [1], and WARBM type 4 (MIM: 615663) by mutations in the TBC1 Domain Family Member 20 (TBC1D20; MIM: 611663) [5]. Of these, $R A B 3 G A P 1$ mutations are by far the most common and approximately account for $40 \%$ of WARBM cases [5].

RAB3GAP1 contains 24 exons and encodes the catalytic subunit of a Rab GTPase activating protein (GAP) that has specificity for the Rab3 subfamily (i.e. RAB3A, RAB3B, RAB3C, and RAB3D) [6]. The catalytic activity of RAB3GAP1 is attributed to its C-terminus (i.e. codons 601-981) [7]. Rab3 proteins are required for normal eye and brain development [8], proper exocytosis of neurotransmitters [9] and hormones [10], and also are imperative for other neurodevelopmental processes such as proliferation, migration, and differentiation of neuronal cells $[11,12]$. Rab3A is the most common isoform in the human brain playing a pivotal role in neurotransmitter release and synaptic plasticity of RAB3GAP, which, in turn, converts active Rab3-GTP to inactive Rab3-GDP [13].

One of the major obstacles for clinicians presented with patients with a combination of different symptoms-e.g. postnatal growth retardation, microcephaly, microphthalmia, and cataracts-is to make a differential diagnosis [14]; however, using some advanced technologies such as wholeexome sequencing (WES), to some extent, has addressed these concerns and helped to make as precisely as diagnosis.

Although different studies have been conducted to investigate into the genotype-phenotype correlation in WARBM patients, yet this still remains blanketed in mystery in different populations. To fill this gap, we describe the clinical characteristics of two Iranian patients with WARBM type 1 . This study may provide insight into the pathogenesis and clinical spectrum and facilitate early diagnosis of this syndrome.

\section{Case presentation}

Two patients-a 5-year-old female and a 4.5-year-old male-were referred to our department due to postnatal microcephaly and ocular manifestations including congenital cataract, microphthalmia, and optic atrophy. Pregnancies were uneventful; the anthropometric data (e.g. weight, length, and head circumference) in their first month of life were reported normal. No contractures were detected in both patients, while mild facial hypertrichosis was evident and limited to the temporal areas of their faces. Their parents were phenotypically normal and also consanguineous. The mothers (II.1 and II.3) had not any confrontation with infection, radiation, or even drug consumption during their pregnancies.

All of the patients' clinical information and medical history were collected at the Department of Medical Genetics, Tarbiat Modares University, Tehran, Iran. Detailed patients' history including disease onset, symptoms, progression, and also family history were gathered at this department. In addition to the traditional biochemistry tests, the patients were subjected to karyotyping using standard procedures to exclude any detectable chromosomal abnormalities. Neuroimaging was carried out using non-contrast brain magnetic resonance imaging (MRI).

\section{Patient 1}

The proband (III.1), a 5-year-old female, was born through normal vaginal delivery at 34 weeks of gestation without any complications. Her birth weight, length, and head circumference $(\mathrm{HC})$ were reported normal. The motor developmental delay was firstly noted after the age of 6 months. After this age, the patient was not able to roll over or even sit on her own. On top of that, she had a severe speech and cognition delay. No seizure was reported by the parents until examination time. Cataract surgery was performed on the patient at the age of 4 years.

Physical and neurologic examination at the age of 5 years revealed a severe head lag, axial hypotonia, sparse voluntary movements, and peripheral spasticity in addition to severe speech and intellectual disability. Head circumference was measured $46 \pm 0.2 \mathrm{~cm} \quad(<-3$ SD) showing postnatal progressive microcephaly. From skeletal points of view, mild thoracolumbar scoliosis (Fig. 1a) and cortical thumb (Fig. 1b) were evident. Some craniofacial features-e.g. large low-set ears, congenital bilateral cataract, microphthalmia, and ptosis (Fig. 1b, c, $\mathrm{d}$-were also apparent. No obvious abnormality was found regarding the patient's feet (Fig. 1e).

Brain MRI without contrast including T1-W, T2-W, and FLAIR sequences was performed on the proband at the age of 5 years and revealed agenesis of the corpus callosum, cerebral white matter hypomyelination as diffuse bilateral abnormal signal changes in subcortical, deep, and periventricular white matter areas, cortical thickening especially in the frontal area, subcortical band 

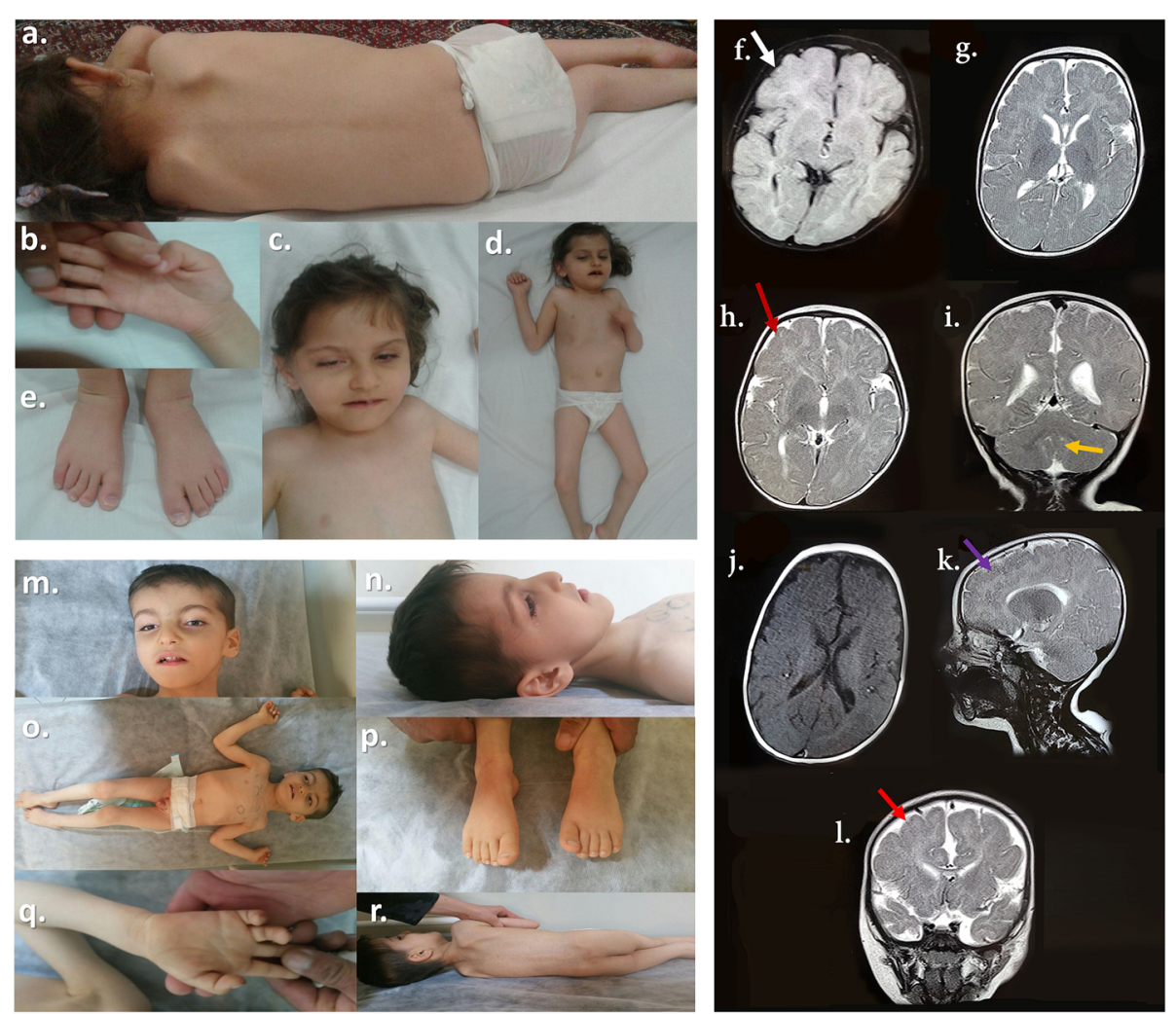

Fig. 1 A summary of important patients' clinical features. The proband (III.1) shows mild thoracolumbar scoliosis and hypertrichosis of the upper back (in the cervical region) (a), the cortical thumb and no contractures (b), mild unilateral ptosis of the right eye and low anterior hairline limited to the temporal sides $(\mathbf{c}, \mathbf{d})$, and normal feet fingers and toes (e). Selected brain MRI sequences of the index case (III.1) at the age of 5 years: Axial T1-W and coronal T2-W images reveal pachygyria in the frontal area (white arrow) and widened Sylvian fissures (f), axial and coronal T2-W images demonstrate diffuse abnormal signal changes in deep and subcortical white matter areas and cortical thickening especially in frontal area (g-i; red and yellow arrows), axial T1-W image illustrates a hypomyelination pattern of deep white matter with partial myelination of LLIC (Lower Limb of Internal Capsule) (j), sagittal T2-weighted MRI scan casts light on enlarged ventricles and agenesis of the corpus callosum and subcortical band heterotopia (k; purple arrow), coronal T2-weighted shows hypomyelination in the pre-dentate white matter of the cerebellum (li; thick red arrowhead). The second patient (III.4) manifested some important clinical features as in downturned corners of the mouth, wide nasal bridge, relatively short nose, mild micrognathia, a mild prominent forehead with bitemporal hollowing $(\mathbf{m})$, bilateral low-set prominent ears, low anterior hairline in lateral sides, and prominent antitragus (n), limb spasticity and hypogenitalism with micropenis and bilateral cryptorchidism (o), overlapping feet toes $(\mathbf{p})$, normal fingers and palm $(\mathbf{q})$, and mild scoliosis and hypertrichosis of the upper back (r)

heterotopia in the anterior part of the brain, and hypomyelination in the pre-dentate white matter of the cerebellum (Fig. 1f-l). Similar findings were obtained for the III.4. Further examinations showed no other abnormality using echocardiography, abdominal ultrasonography, and further skeletal investigations-i.e. examination for osteopetrosis, kyphoscoliosis, osteopenia, and growth hormone deficiency. Besides, using auditory brainstem response $(\mathrm{ABR})$ and pure tone otoacoustic emissions (OAEs) testing, no hearing impairment was detected in the patient.

Hematological examination, thyroid, liver, and renal function tests, serum calcium, ammonia, lactate, pyruvate, and TORCH studies (serum anti-toxoplasmosis, rubella, cytomegalovirus, and herpes virus IgG and IgM antibodies) were all reported normal. Karyotyping did not reveal any detectable chromosomal abnormalities
(Additional file 1). In Table 1, we summarized some of the important clinical findings in this patient, compared to different types of WARBM disorder and Martsolf syndrome (MIM: 212720).

\section{Patient 2}

The second case (III.4) was a 4.5-year-old male born through a normal vaginal delivery without any complications. He had normal birth weight and length and his birth $\mathrm{HC}$ was measured as $31 \pm 0.2 \mathrm{~cm}$ (7th centile). No seizures were recorded in the patient's medical history. According to the medical history, the motor developmental delay was first suspected after the age of 8 months. After this period, the patient was not able to roll over or sit down without any assistant. Furthermore, speech delay and cognitive disability were also noted after 1 year of age. Until the age of 4.5 years, no serious 
Table 1 summary of the important clinical features of the patients. All clinical features are compared to the different types of Warburg Micro Syndrome (WARBM) and Martsolf syndrome

\begin{tabular}{|c|c|c|c|c|c|c|c|c|c|}
\hline Group & Subgroup & Clinical Feature & III.1 & III.4 & WARBM1 & WARBM2 & WARBM3 & WARBM4 & $\begin{array}{l}\text { Martsolf } \\
\text { syndrome }\end{array}$ \\
\hline $\begin{array}{l}\text { aGe at } \\
\text { examination }\end{array}$ & Age (year) & - & 5 & 4.5 & - & - & - & - & - \\
\hline $\begin{array}{l}\text { Intellectual } \\
\text { disability }^{\mathrm{a}}\end{array}$ & - & - & STP & STP & STP & S & STP & S & MTS \\
\hline \multirow[t]{4}{*}{ Growth } & Height & Short stature & NR & NR & + & + & + & + & + \\
\hline & & Height less than 5th percentile & - & - & + & + & + & + & + \\
\hline & & postnatal growth retardation & + & + & + & + & + & + & - \\
\hline & Weight & abnormal Weight & - & - & - & - & - & - & + \\
\hline \multirow[t]{25}{*}{ Head \& neck } & Head & Brachycephaly & - & - & - & + & + & + & + \\
\hline & & Microcephaly & + & + & + & + & + & + & + \\
\hline & Face & Micrognathia & + & + & + & - & + & - & + \\
\hline & & Abnormal philtrum & + & + & - & - & + & - & + \\
\hline & Ears & Prominent antitragus & + & + & - & - & - & - & + \\
\hline & & Posteriorly rotated ears & - & - & - & - & - & - & + \\
\hline & & Large ears & + & + & + & + & + & + & - \\
\hline & Eyes & Cataracts & + & + & + & + & + & + & + \\
\hline & & Epicanthal folds & - & - & - & - & - & - & + \\
\hline & & $\begin{array}{l}\text { Downslanting palpebral } \\
\text { fissures }\end{array}$ & - & - & - & - & - & - & + \\
\hline & & Microphthalmia & + & + & + & + & + & + & - \\
\hline & & Microcornea & NR & NR & + & + & + & + & - \\
\hline & & Optic atrophy & + & + & + & + & - & - & - \\
\hline & & Ptosis & + & + & + & - & - & + & - \\
\hline & & Deep-set eyes & - & - & + & - & - & + & - \\
\hline & & Atonic pupils & - & - & - & + & + & - & - \\
\hline & & Nystagmus & - & - & - & - & + & - & - \\
\hline & Nose & Short-nose & + & + & - & + & + & - & - \\
\hline & & prominent nasal root & + & + & - & + & + & + & - \\
\hline & & Wide nasal bridge & - & + & - & - & - & + & - \\
\hline & Mouth & High palate & - & - & - & - & - & - & + \\
\hline & & $\begin{array}{l}\text { Downturned corners of the } \\
\text { mouth }\end{array}$ & - & + & - & - & + & - & - \\
\hline & & soft cleft palate & - & + & + & - & - & - & - \\
\hline & Teeth & Malaligned teeth & - & + & - & - & - & - & + \\
\hline & & $\begin{array}{l}\text { Prominent secondary alveolar } \\
\text { ridges }\end{array}$ & - & - & - & - & + & - & - \\
\hline \multirow[t]{2}{*}{ Cardiovascular } & Heart & Cardiomyopathy & - & - & - & - & - & - & + \\
\hline & & Cardiac Failure & - & - & - & - & - & - & + \\
\hline \multirow[t]{2}{*}{ Respiratory } & Infection & Recurrent respiratory infections & - & - & - & - & - & - & + \\
\hline & Airway & Tracheomalacia & - & - & - & - & - & - & + \\
\hline \multirow[t]{3}{*}{ Chest } & Breasts & Prominent nipples & - & - & - & - & - & - & + \\
\hline & & Widely spaced nipples & - & - & - & - & + & - & - \\
\hline & Ribs & Pectus carinatum/ excavatum & - & - & - & - & - & - & + \\
\hline Abdomen & Gastrointestinal & Feeding problems & NR & NR & - & - & - & - & + \\
\hline \multirow[t]{2}{*}{ Genitourinary } & Male & Small penis & ND & + & + & - & - & - & + \\
\hline & & Hypogenitalism & + & + & + & + & + & + & - \\
\hline
\end{tabular}


Table 1 summary of the important clinical features of the patients. All clinical features are compared to the different types of Warburg Micro Syndrome (WARBM) and Martsolf syndrome (Continued)

\begin{tabular}{|c|c|c|c|c|c|c|c|c|c|}
\hline Group & Subgroup & Clinical Feature & III.1 & III.4 & WARBM1 & WARBM2 & WARBM3 & WARBM4 & $\begin{array}{l}\text { Martsolf } \\
\text { syndrome }\end{array}$ \\
\hline & & $\begin{array}{l}\text { Scrotal hypoplasia/ Small } \\
\text { testes }\end{array}$ & ND & + & - & + & + & - & - \\
\hline & & Cryptorchidism & ND & + & + & - & - & + & + \\
\hline & Female & Hypoplasia of labia majora & + & - & - & + & + & - & - \\
\hline \multirow[t]{8}{*}{ Skeletal } & \multirow[t]{3}{*}{ Limbs } & Contractures & - & - & - & + & + & + & - \\
\hline & & Distal limb contractures & - & - & - & - & + & - & - \\
\hline & & Joint hypermobility & - & - & + & - & - & - & - \\
\hline & \multirow[t]{3}{*}{ Hands } & Finger joint laxity & - & - & - & - & - & - & + \\
\hline & & Joint hypermobility & - & - & + & - & - & - & - \\
\hline & & Cortical thumbs & + & - & - & - & + & - & - \\
\hline & \multirow[t]{2}{*}{ Feet } & $\begin{array}{l}\text { Deformities of metatarsal } \\
\text { bones }\end{array}$ & - & - & + & - & - & - & - \\
\hline & & Overlapping toes & + & + & + & + & - & - & - \\
\hline \multirow[t]{4}{*}{ Skin, nails, \& hair } & Nails & Abnormal nails & - & - & - & - & - & - & + \\
\hline & \multirow[t]{3}{*}{ Hair } & Facial hypertrichosis & + & + & + & - & - & - & - \\
\hline & & Low anterior hairline & + & + & - & + & + & + & - \\
\hline & & Hypertrichosis of upper back & + & + & - & - & + & - & - \\
\hline \multirow[t]{19}{*}{ Neurologic } & \multirow{9}{*}{$\begin{array}{l}\text { Central and peripheral Nervous } \\
\text { Systems }\end{array}$} & Severe intellectual disability & + & + & + & + & + & + & + \\
\hline & & Truncal hypotonia & NR & NR & - & - & + & - & - \\
\hline & & Hyperreflexia & NR & NR & + & - & - & - & - \\
\hline & & Limbs hypotonia & + & + & + & - & - & - & - \\
\hline & & Axial hypotonia & + & + & - & + & - & + & - \\
\hline & & White matter signal changes & + & + & - & - & - & - & - \\
\hline & & $\begin{array}{l}\text { Spastic diplegia to } \\
\text { quadriplegia }\end{array}$ & + & + & - & + & + & + & - \\
\hline & & $\begin{array}{l}\text { Increased deep tendon } \\
\text { responses }\end{array}$ & - & - & - & - & + & - & - \\
\hline & & Ankle clonus & - & - & - & - & + & - & - \\
\hline & \multirow[t]{10}{*}{ Neuroimaging Findings } & Cerebral malformations & + & + & + & - & - & + & - \\
\hline & & $\begin{array}{l}\text { Hypoplasia of the corpus } \\
\text { callosum }\end{array}$ & - & + & + & + & + & + & - \\
\hline & & $\begin{array}{l}\text { Agenesis of the corpus } \\
\text { callosum }\end{array}$ & + & - & + & + & - & - & - \\
\hline & & Cerebellar hypoplasia & + & + & + & - & - & + & - \\
\hline & & Wide Sylvian fissures & + & + & - & + & - & - & - \\
\hline & & Increased subdural space & + & + & - & + & - & - & - \\
\hline & & Enlarged ventricles & + & + & - & - & + & + & - \\
\hline & & Pachygyria & + & + & + & - & - & - & \\
\hline & & Polymicrogyria & - & - & - & + & + & + & - \\
\hline & & Mega cisterna Magna & - & - & - & - & - & + & - \\
\hline
\end{tabular}

Abbreviations: STP severe-to-profound intellectual disability, S severe, MTS moderate-to-severe intellectual disability, NR Not Reported or detected at the age of examination, ND Not defined or not-appropriate value for the specific gender

${ }^{a}$ The intelligence quotient (IQ) scores were used to classified the intellectual disability (ID) into mild (IQ 50/55 to 70 ), moderate (35/40 to 50/55), severe $(20 / 25$ to $35 / 40)$, and profound $(<25)$

medical evaluation had been performed for a better diagnosis.

Clinical examinations at the age of 4.5 years showed quite similar findings in this patient such as noticeably delayed gross motor development with no head control, axial hypotonia, profound intellectual and speech abilities, and four limb spasticity. HC was measured $49 \pm$ $0.2 \mathrm{~cm}(<-3 \mathrm{SD})$ at the age of examination, indicating 
postnatal microcephaly. The general examination demonstrated a soft cleft palate, downturned corners of the mouth, wide nasal bridge, relatively short nose, mild micrognathia, a mild prominent forehead with bitemporal hollowing, bilateral low-set prominent ears, hypogenitalism with micropenis and bilateral cryptorchidism, and mild scoliosis (Fig. 1m-q). As a prominent clinical feature, the patient showed hypertrichosis of the upper back (Fig. 1r). Ocular abnormalities such as cataract, nasolacrimal duct obstruction, and bilateral microphthalmia were also detected at the time of examination.

Brain MRI without contrast-including T1-W, T2-W, and FLAIR sequences-performed at this age and showed the hypoplasia of the corpus callosum, cerebral white matter hypomyelination, cortical thickening (especially in the frontoparietal area), widened Sylvain fissure, enlarged ventricles, and hypomyelination in the white matter of the cerebellum. Electrocardiogram, bone scan, neonatal TORCH studies, and basic metabolic panel tests were all normal. G-banded chromosome analysis revealed a normal male karyotype as well (Additional file 1).

\section{Genetic findings and determination of allele frequency of} the candidate variants

Due to the heterogeneity of neurodevelopmental disorders [15], we subjected the patients, parents, and also a healthy sibling to paired-end WES screening to detect the possible underlying genetic factor. WES was performed based on the previous studies [16-18]. The mean depth of coverage was around 100× and approximately $98 \%$ of targeted regions were covered (Additional file 2). The overlapped filtering strategy was used to detect the common variants among the patients and the parents (Fig. 2a, b). The filtering steps were carried out according to the previous studies [19]. In total, 780,385 common SNV and Indel variants among all the 7 genotyped individuals (are shown by an asterisk $\left(^{*}\right)$ in Fig. 2a) were detected. By doing the overlapped filter strategy, 4297 variants were detected. By excluding the synonymous, low-functional profile, and variants with mutation allele frequencies (MAF) greater than $1 \%$ in publicly available databases-e.g dbSNP150 [20], 1000 Genomes Project [21], Exome Sequencing Project [22], and gnomAD [23] or ExAC databases [24]—only a.

II

III

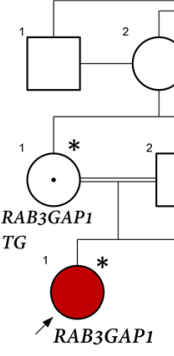

GG

c.

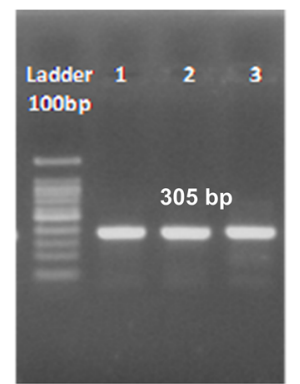

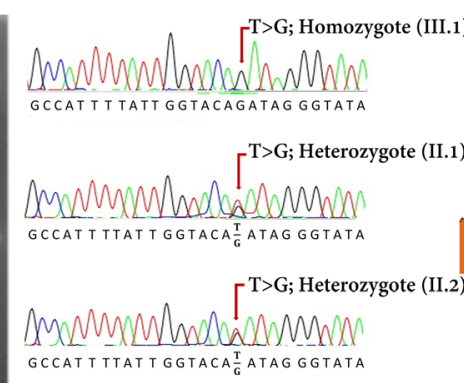

b.

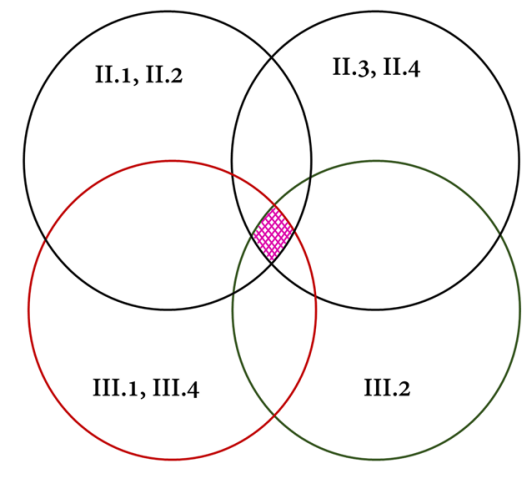

d.

Intolerant Neutral Tolerant

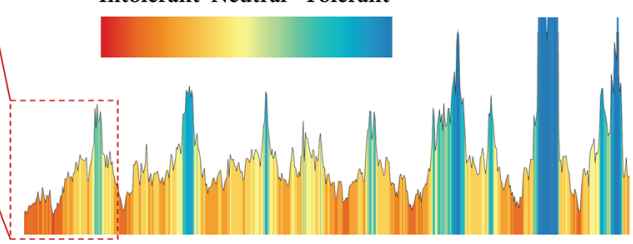
c. 151

Fig. 2 c.151-5 T > G was identified using whole-exome and Sanger sequencing in the family. a Pedigree of the family in which the carriers and patients are indicated as TG and GG, respectively. The proband (III.1), III.4, parents (II.1, II.2, II.3, and II.4), and III.2 were subjected to WES (all are shown by an asterisk $\left(^{*}\right)$ ). In this pedigree, white symbols: unaffected who were homozygous for wild-type allele; red symbol: affected and homozygous for the variant; squares: males; circles: females; parallel lines: consanguineous marriage. The status of RAB3GAP1 variation is shown for each participant, in which, TG: heterozygote for novel variant and wild-type allele, and GG: homozygote for the variant. b Overlapped filtering strategy. The red zone indicates the remained variants for further analysis that are presented as a homozygote in 2 affected members (III.1 and III.4) but not in other individuals. These variants have been seen in heterozygous in other individuals (II.1, II.2, II.3, II.4, and III.2). The purple zone shows the common variants between all individuals. c Chromatograms showing nucleotide sequences of c.151-5T>G of RAB3GAP1. In this figure, the size of the sequenced DNA region is shown using 2\% gel electrophoresis, in which 1 denotes the patient sample, 2 and 3 show the mother and father samples, respectively. $\mathbf{d}$ MetaDome was used to identify the intolerant regions (surrounding the c.151-5 T > G variant) in RAB3GAP1. As depicted, the affected nucleotide/residue is located in a highly intolerant region 
three variants were identified in MAP 3 K19, RAB3GAP1, and XIRP2 genes, respectively. Different tools, e.g. SIFT [25], Polyphen-2 [26], MutationTaster [27], and Provean [28] were also executed to evaluate the pathogenicity of the identified variants (Table 2). To investigate the cosegregation analysis, the primers were designed using Primer3.0 online tool [29] (Additional file 3) and the variants were subjected to Sanger sequencing (Additional file 4; Fig. 2c). The reference sequences of NM_001199144.1 for XIRP2, NM_001018044.2 for MAP3K19, and NM 012233.2 for RAB3GAP1 were used based on the human genome assembly GRCh37 (hg19).

In the nucleotide level, conservational analyses using ConSurf [30] and '2-Way Pseudogene Annotation Set' of UCSC [31] showed that c.151 T is located in a highly conserved region (Fig. 2d). On the other hand, c.9835 $\mathrm{T}>\mathrm{C}$, p.(Ser3279Pro) in XIRP2 and c.332 T $>\mathrm{C}$, p.(Ile111Thr) in MAP3K19 were categorized as nonconserved variants. MetaDome [32] was also used to visualize and confirm the data (Additional file 4).

To exclude the variants with low detrimental effects, we determined the allele frequency in 300 healthy and ethnically matched controls. For this purpose, we used PCR-Restriction Fragment Length Polymorphism (PCRRFLP) and also tetra-primer Amplification Refractory Mutation System-PCR (tetra-primer ARMS-PCR). Using PCR-RFLP (Additional file 2), the allele frequency of c. $9835 \mathrm{~T}>\mathrm{C}$ variant in XIRP2 was determined 0.031, moreover, a homozygous individual was detected in healthy controls (Additional file 5). c. $9835 \mathrm{~T}>\mathrm{C}$ variant causes a loss of the XapI restriction site. The PCR products were digested with XapI and run on $2 \%$ agarose gel to assess whether the variant allele existed or not. This data was in line with the findings of Iranome [33] (allele frequency: 0.01063), suggesting that this variant cannot be considered pathogenic, so we excluded the variant from further investigations.

To determine the allele frequency of c. $332 \mathrm{~T}>\mathrm{C}$ in MAP3K19 and c.151-5 T>G in RAG3GAP1 in 300 healthy ethnicity-matched controls, tetra-primer ARMSPCR was performed (Additional File 6; Fig. 3a). No homozygote/heterozygote was identified among 300 samples; consistently, Iranome and gnomAD verified these findings. The primers and size of expected bands in each reaction are depicted in Additional file 3. The procedure was carried out according to the previous study [34] (Additional file 2).

\section{c.151-5T> $\mathrm{G}$ affects splicing}

Using 'MaxEnt Acceptor site algorithms' of Human Splicing Finder V.3.1 (HSF; https://hsf.genomnis.com/) [35], we showed that c.151-5 T > G may alter the wildtype acceptor site, thus can most probably affect splicing. This finding was confirmed by Berkeley Drosophila
Genome Project (BDGP; https://www.fruitfly.org) [36] and NetGene2 (http://www.cbs.dtu.dk/services/ NetGene2) [36] tools. To predict if the variant can affect the cryptic splice-site activation and exon skipping, CRYP-SKIP (https://cryp-skip.img.cas.cz/) program [36] was used; these data showed that the c.151-5 T > G variant can increase the probability of cryptic splice site activation. To confirm, we used RT-PCR on the samples of the proband (III.1) and her parents (II.1 and II.2). The PCR products were then sequenced using Sanger sequencing with same primers (Additional file 3). The results revealed that the novel variant can activate a cryptic splice site within intron 3 of RAB3GAP1. This can change the splicing process of the RAB3GAP1 gene by adding 4 nucleotides between exons 3 and 4 . In sum, this leads to the losing 930 amino acids (Fig. 3b-d).

The relative expression levels of RAB3GAP1 in the peripheral blood cells of the index case (III.1), parents (II.1 and II.2), and also a control/normal individual (III.3) were compared. The normal individual (control) was initially screened for the c.151-5 T $>\mathrm{G}$ variant and he was negative for this variant. RT-qPCR was performed using SYBR ${ }^{\circ}$ Premix Taq ${ }^{\text {tw }}$ II (TAKARA, Tokyo, Japan) on an ABI StepOne Sequence Detection System (Applied Biosystems, Foster City, USA). Each experiment was performed in triplicate and expression data were normalized to $A L B$ (albumin) as an internal reference gene. Primer sequences are put forward in Additional file 3. The $2^{-\Delta \Delta \mathrm{Ct}}$ method was used for quantification by comparing the Ct values $[37,38]$. The RT-qPCR analysis showed that the RAB3GAP1 expression was significantly decreased in the proband (Fig. 3e, f) and also demonstrated that only a trivial amount of $R A B 3 G A P 1$ expression remained as a result of homozygous c.151-5 T > G splice site variant. This underscores the potential pathogenic effect of this novel variant (Fig. 3b, c). On the other hand, the decreased expression of RAB3GAP1 was determined in parents who were heterozygous for the novel variant (Fig. 3e, f).

To show whether the impaired genes make a contribution to brain development/function, we explored the regional expression of MAP3K19 and RAB3GAP1 in the normal adult human brain. For this purpose, we used microarray data (Affymetrix Exon 1.0 ST) from human post-mortem brain tissue collected by the UK Human Brain Expression Consortium [39]. To confirm, we also used the data available in the Allen Mouse Brain Atlas $[40,41]$. To show how the expression of Map3k19 (MGI: 1203481) and Rab3gap1 (MGI: 2445001) changes during brain development, we utilized the 'Gene Expression Database (GXD) Project' [42] of MGI which is an international database resource for the laboratory mouse. The results showed that Map3k19 has a subtle expression during brain development that per se cannot justify a broad range of brain abnormalities in the patients. 


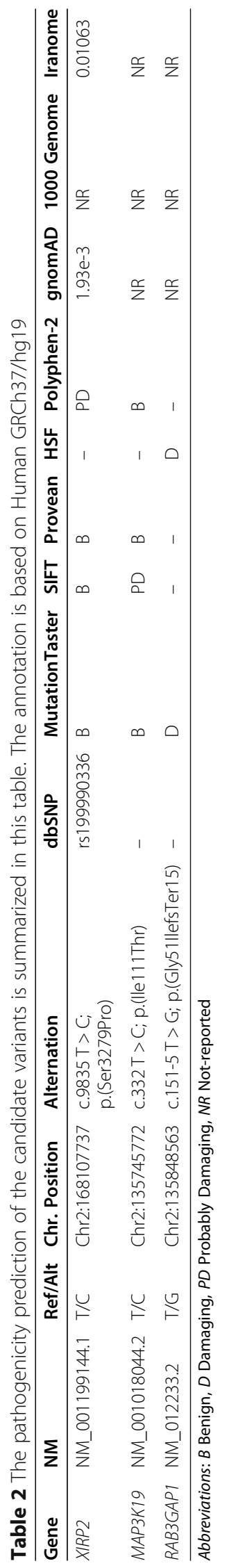




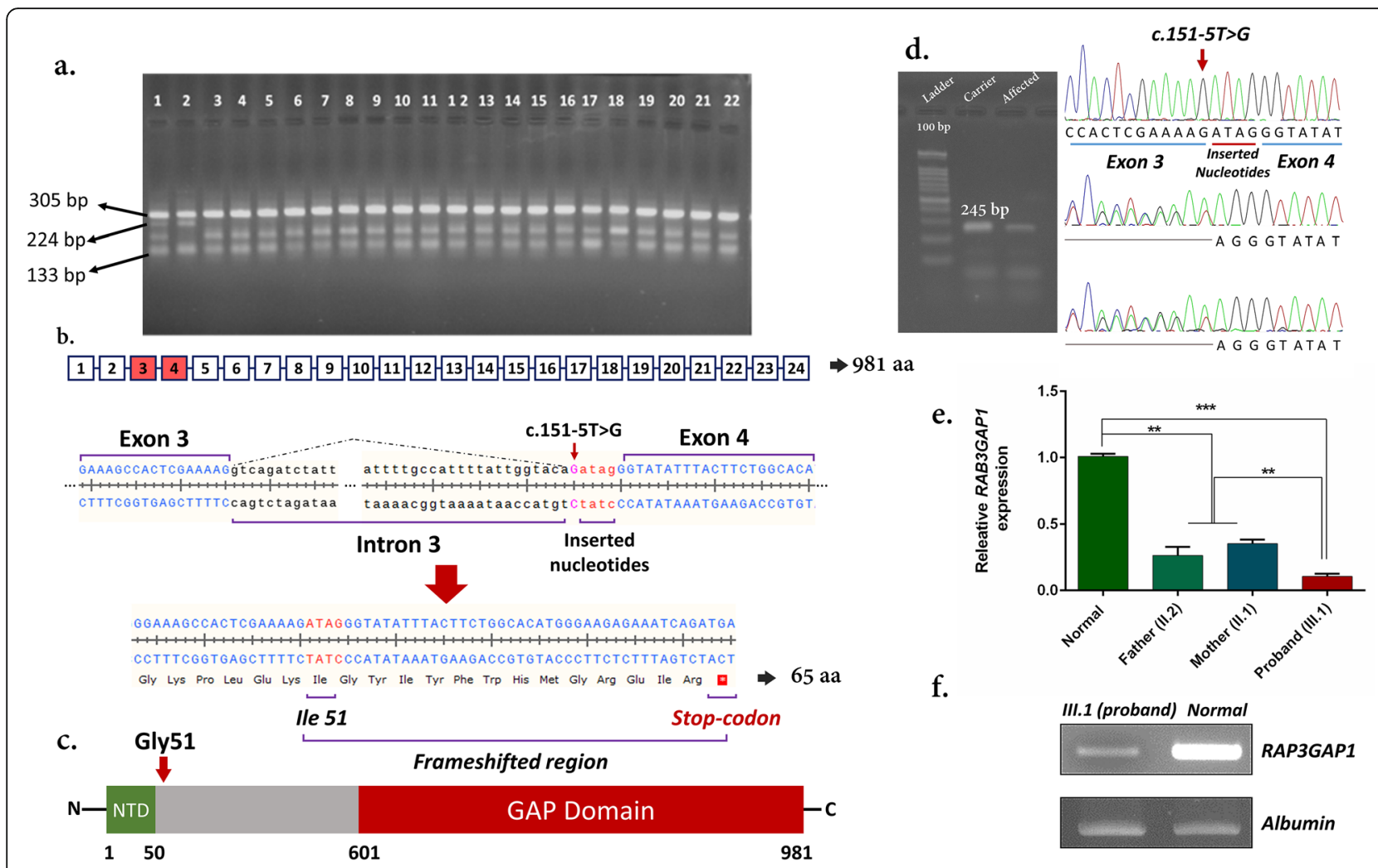

Fig. 3 Determining the allele frequency of c.151-5 T > G variant and expression of RAB3GAP1. a a tetra-primer ARMS-PCR was used to determine the allele frequency of c.151-5 T > G variant on the RAB3GAP1 gene. In this figure, well 1: heterozygous for wild-type allele, well 2: Homozygous for c.151-5 T> G variant, well 3: homozygous for the wild-type allele. $\mathbf{b}$ a schematic figure shows the 24 exons of RAB3GAP1 and also the effects of c.151-5 T > G on splicing. The c.151-5 T > G inserts 4 nucleotides that in turn cause frameshifting and generating the truncated protein. $\mathbf{c}$ Schematic of the RAB3GAP1 protein showing the Rab3 GAP domain (red) and the conserved N-terminal region of RAB3GAP1 (NTD; green). $\mathbf{d}$ RTPCR was performed on RNA samples of patients and carriers (Parents). Chromatograms revealing nucleotide sequences of c.151-5 T > G of RAB3GAP1. In this figure, the size of the sequenced CDNA region is shown using $2 \%$ gel electrophoresis. e and $\mathbf{f}$ Relative expression levels of RAB3GAP1 in peripheral blood cells of the patient (proband), parents, and normal individual (III.3) shows a significant reduction in the patient's mRNA level. III.3 prescreened for c.151-5 T > G variant. The experiment was performed in triplicate and ALB (albumin) was used as an internal reference gene. The data are presented as the mean \pm standard deviation (SD) and the statistical significance was evaluated by Student's t-test. Statistical analysis was performed by SPSS version 24.0 (SPSS Inc., Chicago, IL, USA) and GraphPad Prism v.8.0 (GraphPad, San Diego, USA). Pvalues $<0.05$ were considered to be statistically significant. ${ }^{* *} P<0.01,{ }^{* * *} P<0.001$

On the other hand, the aberrant expression of the RAB3GAP1 was significantly expected to be associated with the diverse brain impairments in WARBM disorders (Additional file 7).

\section{Discussion and conclusions}

WARBM is a heterogeneous group of autosomal recessive disorders that can be resulted from loss-of-function mutations in RAB3GAP1/2, RAB18, and TBC1D20 [43, 44]. These genes encode the 'RAB proteins' that mainly function as molecular switches [45]; they can also regulate membrane trafficking in a spatially and temporally restricted manner [46]. GAP proteins can regulate RAB protein cycling [47], e.g., RAB3GAP1 (catalytic subunit) and RAB3GAP2 (non-catalytic subunit) make a heterodimeric enzyme complex, which has GAP activity that is specific for the RAB3 family of protein. This heterodimeric enzyme complex hydrolyzes GTP into GDP and also modulates the $\mathrm{Ca}^{2+}$ mediated exocytosis of hormones and neurotransmitters [48].

Most of the mutations in RAB3GAP1-in WARBM patients-are predicted to result in stop-codons and are distributed throughout the coding region from codons 89 to 934 [3]. In a nutshell, all kinds of these mutations can lead to a truncated protein either before or within the regions that are important for catalytic activity [3] (Fig. 3c). This also can explain why there are some phenotypic variations between the WARBM with different mutations in RAB3GAP1. Rab3gap1 mutant and knockout mice models have previously been generated, while none of them embraced all major clinical features of WARBM [49]. In mice, disruption of Rab3gap1 results in increased short-term depression and also paired-pulse facilitation at CA1 hippocampal neurons [49]. On the other 
hand, human case reports have successfully covered a broad range of phenotypes caused by mutations in RAB3GAP1.

The majority of WARBM cases, similar to our case, are resulted from consanguineous marriages [50]. Here, after performing WES, as a final candidate, we identified a homozygous pathogenic variant c.151-5 T $>$ G; p.(Gly51IlefsTer15) in the intron 3 of the RAB3GAP1 gene, justifying the phenotypes that were in association with autosomal recessive WARBM type 1 . Data from in silico analyses and also RT-PCR showed that c.151-5 $\mathrm{T}>\mathrm{G}$ can activate a cryptic splice site in intron 3 . This led to the addition of four nucleotides upstream of exon 4 , which in turn changes the mRNA reading frame; this causes a loss of 971 codons, due to the premature stop codon. Moreover, RT-qPCR data showed that the expression of RAB3GAP1 harboring c.151-5 $\mathrm{T}>\mathrm{G}$ variant was significantly decreased in the patients than the normal individual (III.3). Although the parents showed lower mRNA levels than the control group, they did not show any symptoms of the disease. This can per se confirm that the low amount of RAB3GAP1 protein is also sufficiently functional. There are two fates for mRNAs containing premature termination codons: nonsensemediated mRNA decay (NMD) [51] or translation to truncated proteins [52]. NMD is an evolutionarily conserved quality control pathway in eukaryotic cells that is responsible for inspecting mRNA for any possible errors, so eliminating any error-containing transcripts and controlling the amount of non-mutated transcript in the transcriptome (reviewed in [53]). Therefore, NMD leads to loss-of-function allele [51]; herein, RT-qPCR data supported the NMD hypothesis. Axiomatically, RAB3GAP1 protein that embraces p.(Gly51IlefsTer15) will be destroyed or be malfunctioned. It was previously suggested that pathogenic mutations in RAB3GAP1 with WARBM type 1 were mostly frameshift, nonsense, and splicing mutations causing complete loss of function [54]; Our data underscore this hypothesis as well. We also reclassified the variant based on the American College of Medical Genetics and Genomics (ACMG) variant interpretation guideline [55] into the 'Pathogenic' variant.

Homozygous and compound heterozygous mutations in human RAB3GAP1 cause WARBM type 1 and also a somehow milder phenotype called Martsolf syndrome [6]. These two disorders are genetically heterogeneous and comprise a phenotypic spectrum described as "RAB18 deficiency" based on molecular etiology [6]. Mutations in RAB3GAP2 can cause Martsolf syndrome. To exclude this syndrome, we meticulously compared the phenotypes of the patients in Table 1. Indeed, patients with Martsolf syndrome manifest different degrees of ID-from mild to severe-while WARBM patients show often severe-to-profound ID with no or even very limited communication skills. Spastic quadriplegia has been reported in WARBM, whereas in Martsolf syndrome, spasticity is limited to lower limbs [56]. Molecularly, mutations reported in association with WARBM are mostly predicted to result in NMD and/or loss-of-protein-function [57]. On top of that, we also found that some clinical features, e.g. hypoplasia of the corpus callosum, microcephaly, and developmental delay, can be observed in other types of WARBM in addition to Martsolf syndrome. Besides, based on some common clinical features among these patients and Martsolf syndrome, e.g. prominent antitragus, malaligned/misaligned teeth, and small penis, we suggest using the "RABopathies" term that can efficiently cover all clinical features between different types of WARBM and Martsolf syndrome.

We also introduced some novel manifestations of WARBM type 1 including abnormal philtrum, prominent antitragus, downturned corners of the mouth, malaligned teeth, scrotal hypoplasia, low anterior hairline in temporal areas, hypertrichosis of upper back, spastic diplegia to quadriplegia, and signal changes in deep and subcortical white matter areas. Perhaps, one of the most recognizable features of WARBM is the brain phenotype. Herein, clinical examinations showed that the patients (III.1 and III.4) manifested postnatal microcephaly which has been observed in most cases of the Martsolf syndrome and the WARBM. Developmental milestones in two patients were compatible with severe ID (Table 1).

The present study demonstrated that a novel homozygous variant in RAB3GAP1 is the main cause of WARBM type 1 disorder characterized by abnormal philtrum, prominent antitragus, downturned corners of the mouth, cerebral white matter signal changes, malaligned teeth, scrotal hypoplasia, low anterior hairline, hypertrichosis of upper back, spastic diplegia to quadriplegia as novel manifestations in WARBM type 1. We also coined the term "RABopathies" that can cover the overlapped clinical features among WARBM and Martsolf syndrome. Moreover, we reconfirmed that the WES can properly detect underlying genetic factors contributing to the neurodevelopmental disorder that show a great phenotype overlapping. This study also provides important information to guide genetic counseling and expands the genotype-phenotype spectrum of WARBM type 1.

\section{Abbreviations}

ACMG: American College of Medical Genetics and Genomics; GXD: Gene Expression Database; HC: Head circumference; MRI: Magnetic resonance imaging; PCR-RFLP: PCR-Restriction Fragment Length Polymorphism; NMD: Nonsense-mediated mRNA decay; RT-PCR: Reverse transcription-PCR; RT-qPCR: Quantitative reverse transcription-PCR; Tetra-primer ARMSPCR: Tetra-primer Amplification Refractory Mutation System-PCR; WARBM: Warburg micro syndrome; WES: Whole-exome sequencing 


\section{Supplementary Information}

The online version contains supplementary material available at https://doi. org/10.1186/s12883-021-02204-w.

Additional file 1. The karyotypes of the proband (a) and the male affected individual or III.4 (b). The karyotypes did not show any obvious chromosomal changes

Additional file 2. Detailed procedures of Whole-Exome Sequencing and Tetraplex-Amplification refractory mutation system (Tetraple-ARMS-PCR)

Additional file 3. A list of primers that was used in this study

Additional file 4. Sanger sequencing and conservational analysis for the candidate variants in MAP3K19 and XIRP2 genes. a) Chromatograms shed light on the cosegregation of C.332 T > C of MAP3K19 in the family members. b) Chromatograms showing nucleotide sequences of XIRP2 in the regions of c.9835 T > C. c) MetaDome shows that the c.332 T is located in a region with an average conservational profile in MAP3K19 protein. d) In this XIRP2 tolerance landscape, the region harboring the novel missense variant is partially tolerant in comparison with other parts in this protein. The affected region is located in a somehow variable region

Additional file 5. $2 \%$ agarose gel electrophoresis of tetra primer ARMSPCR test products for C.332 T>C in the MAP3K19 gene. In this figure: well 1: 100 bp ladder, well 2: negative PCR test control (no template control; NTC), well 3: Homozygous for the normal allele, well 4: Heterozygous for the wild-type allele. The allele frequency was estimated at 0.031 for the c.332 T > C variant

Additional file 6. A simple and rapid PCR-RFLP assay was used to detect the $\mathrm{C.9835} \mathrm{T}>\mathrm{C}$ variant in the XIRP2 gene. a) This schematic figure shows the generated fragments after digestion with the Xapl restriction enzyme. The variant causes losing the restriction enzyme site. Agarose gel (2.0\%) electrophoresis with ethidium bromide staining following the Xapl digestion of the PCR products is shown. PCR-RFLP results in normal control showing 340, 249, and 130 bp (T/T: wild-type allele); after Xapl digestion, a heterozygous sample will show four distinct bands including 470, 340, 249, and $130 \mathrm{bp}$. The homozygote reveals three distinct bands consisting of 470,249 , and $130 \mathrm{bp}$. b) PCR-RFLP assay was used on the samples in order to show the genotyping. In this figure, 1: heterozygote, 2: patient, 3-14, and 16-22 are wild-type alleles

Additional file 7. a) Gene expression of the RAB3GAP1 is changing in different brain regions as age increases. b) Gene expression of RAB3GAP1 in different brain regions and sexes. c) Boxplots of RAB3GAP1 mRNA expression levels in ten adult brain regions. The expression levels are based on exon array experiments and are plotted on a log2 scale (y-axis) This dataset was generated with Affymetrix Exon 1.0 ST arrays and brain tissue originating from 134 control individuals, collected by the Medical Research Council Sudden Death. The plot also shows significant variation in RAB3GAP1 expression across the ten brain regions analyzed, such that expression is higher in the temporal cortex than in any other region. In this figure, TCTX: temporal cortex, FCTX: frontal cortex, OCTX: occipital cortex, SNIG: substantia nigra, THAL: thalamus, PUTM: putamen, HIPP: hippocampus, CRBL: cerebellar cortex, MEDU: medulla (specifically the inferior olivary nucleus), WHMT: intralobular white matter, and N: number of samples analyzed for each brain region. These figures shed light on RAB3GAP1 expression in the different parts of the brain

\section{Acknowledgments}

We thank the family members for their willingness and contribution.

\section{Authors' contributions}

RK, MG, YR, and DA conceived and planned the experiments. RK carried out the experiments. MG, RK, ER, and GA planned and carried out the data analyses. RK contributed to sample preparation. ER, RK, YR, and GA contributed to the interpretation of the results. ER took the lead in writing the manuscript. ART and ER carried out clinical analyses. All authors provided critical feedback and helped shape the research, analysis, and manuscript. All authors read and approved the final manuscript.

\section{Funding}

The funders provided roles in study design, execution, and data interpretation. This research received specific grants from de recherché du Québec-Santé, Québec, Canada (Grant Number: 269552), and Tarbiat Modares University, Tehran, Iran (Grant Number: TMU.8198.52D).

\section{Availability of data and materials}

The datasets used and/or analyzed during the current study are available from the corresponding author on reasonable request. The variant and pertinent phenotypes caused by a mutation in RAB3GAP1 are accessible at ClinVar (SCV001426394) and Leiden Open Variation Database (LOVD; individual number: 279559).

\section{Declarations}

\section{Ethics approval and consent to participate}

The research protocol was approved by the ethics committee of Tarbiat Modares University, Tehran, Iran under the ethical code of "TMU.8198.52D." The written informed consent was received from each guardian and they also provided a signed written consent to publish all personal and medical details included in this study in addition to the accompanying pictures. Beyond that, all methods were performed in accordance with the relevant guidelines and regulations of the Declaration of Helsinki.

\section{Consent for publication}

The informed consent to publish was obtained from each guardian.

\section{Competing interests}

The authors declare that they have no competing interests.

\section{Author details}

${ }^{1}$ Department of Medical Genetics, Faculty of Medical Sciences, Tarbiat Modares University, Tehran, Iran. ${ }^{2}$ Department of Genetics, Faculty of Biological Sciences, Tarbiat Modares University, Tehran, Iran. ${ }^{3}$ Myelin Disorders Clinic, Pediatric Neurology Division, Children's Medical Center, Pediatrics Center of Excellence, Tehran University of Medical Sciences, Tehran, Iran. ${ }^{4}$ McGill Genome Centre, Montréal, Québec, Canada. ${ }^{5}$ Department of Human Genetics, McGill University, Montréal, Québec, Canada.

Received: 15 November 2020 Accepted: 15 April 2021

Published online: 28 April 2021

\section{References}

1. Bem D, Yoshimura S-I, Nunes-Bastos R, Bond FF, Kurian MA, Rahman F, et al. Loss-of-function mutations in RAB18 cause Warburg micro syndrome. Am J Hum Genet. 2011;88(4):499-507. https://doi.org/10.1016/j.ajhg.2011.03.012.

2. Dursun F, Güven A, Morris-Rosendahl D. Warburg micro syndrome. Pediatr Endocrinol Metab. 2012;25(3-4):379-82. https://doi.org/10.1515/ jpem-2011-0459.

3. Morris-Rosendahl DJ, Segel R, Born AP, Conrad C, Loeys B, Brooks SS, et al. New RAB3GAP1 mutations in patients with Warburg micro syndrome from different ethnic backgrounds and a possible founder effect in the Danish. Eur J Hum Genet. 2010;18(10):1100-6. https://doi.org/10.1038/ejhg.2010.79.

4. Borck G, Wunram H, Steiert A, Volk AE, Körber F, Roters S, et al. A homozygous RAB3GAP2 mutation causes Warburg micro syndrome. Hum Genet. 2011;129(1):45-50. https://doi.org/10.1007/s00439-010-0896-2.

5. Liegel RP, Handley MT, Ronchetti A, Brown S, Langemeyer L, Linford A, et al. Loss-of-function mutations in TBC1D20 cause cataracts and male infertility in blind sterile mice and Warburg micro syndrome in humans. Am J Hum Genet. 2013;93(6):1001-14. https://doi.org/10.1016/j.ajhg.2013.10.011.

6. Aligianis IA, Morgan NV, Mione M, Johnson CA, Rosser E, Hennekam RC, et al. Mutation in Rab3 GTPase-activating protein (RAB3GAP) noncatalytic subunit in a kindred with Martsolf syndrome. Am J Hum Genet. 2006;78(4): 702-7. https://doi.org/10.1086/502681.

7. Fukui K, Sasaki T, Imazumi K, Matsuura Y, Nakanishi H, Takai Y. Isolation and characterization of a GTPase activating protein specific for the Rab3 subfamily of small G proteins. J Biol Chem. 1997;272(8):4655-8. https://doi. org/10.1074/jbc.272.8.4655.

8. Ng EL, Tang BL. Rab GTPases and their roles in brain neurons and glia. Brain Res Rev. 2008;58(1):236-46. https://doi.org/10.1016/j.brainresrev.2008.04.006. 
9. Coppola T, Perret-Menoud V, Lüthi S, Farnsworth CC, Glomset JA, Regazzi R. Disruption of Rab3-calmodulin interaction, but not other effector interactions, prevents Rab3 inhibition of exocytosis. EMBO J. 1999;18(21): 5885-91. https://doi.org/10.1093/emboj/18.21.5885.

10. Tahara S, Sanno N, Teramoto A, Osamura RY. Expression of Rab3, a Rasrelated GTP-binding protein, in human nontumorous pituitaries and pituitary adenomas. Mod Pathol. 1999;12(6):627-34.

11. Iwasaki K, Toyonaga R. The Rab3 GDP/GTP exchange factor homolog AEX-3 has a dual function in synaptic transmission. EMBO J. 2000;19(17):4806-16. https://doi.org/10.1093/emboj/19.17.4806.

12. Khvotchev MV, Ren M, Takamori S, Jahn R, Südhof TC. Divergent functions of neuronal Rab1 1b in Ca2+-regulated versus constitutive exocytosis. J Neurosci. 2003;23(33):10531-9. https:/doi.org/10.1523/jneurosci.23-33-10531.2003.

13. Handley MT, Aligianis IA. RAB3GAP1, RAB3GAP2 and RAB18: disease genes in Micro and Martsolf syndromes. Biochem Soc Trans. 2012;40(6). https://doi. org/10.1042/bst20120169.

14. Shotelersuk V, Desudchit T, Suwanwela N. Postnatal growth failure, microcephaly, mental retardation, cataracts, large joint contractures, osteoporosis, cortical dysplasia, and cerebellar atrophy. Am J Med Genet A. 2003;116(2):164-9. https://doi.org/10.1002/ajmg.a.10067.

15. Dajani DR, Llabre MM, Nebel MB, Mostofsky SH, Uddin LQ. Heterogeneity of executive functions among comorbid neurodevelopmental disorders. Sci Rep. 2016;6(1):36566. https://doi.org/10.1038/srep36566.

16. Gilani N, Razmara E, Ozaslan M, Abdulzahra IK, Arzhang S, Tavasoli AR, et al. A novel deletion variant in CLN3 with highly variable expressivity is responsible for juvenile neuronal ceroid lipofuscinoses. Acta Neurol Belg. 2021:1-12. https://doi.org/10.1007/s13760-021-01655-9.

17. Soleimanipour F, Razmara E, Rahbarizadeh F, Fallahi E, Khodaeian M, Tavasoli AR, et al. A novel missense variant in the LMNB2 gene causes progressive myoclonus epilepsy. Acta Neurol Belg. 2021:1-9. https://doi. org/10.1007/s13760-021-01650-0.

18. Razmara E, Azimi H, Tavasoli AR, Fallahi E, Sheida SV, Eidi M, et al. Novel neuroclinical findings of autosomal recessive primary microcephaly 15 in a consanquineous Iranian family. Eur J Med Genet. 2020;63(12):104096. https://doi.org/10.1016/j.ejmg.2020.104096.

19. Garshasbi M, Mahmoudi M, Razmara E, Vojdanian M, Aslani S, Farhadi E, et al. Identification of RELN variant p.(Ser2486Gly) in an Iranian family with ankylosing spondylitis; the first association of RELN and AS. Eur J Hum Genet. 2020;28(6):1-9. https://doi.org/10.1038/s41431-020-0573-4.

20. Sherry ST, Ward M-H, Kholodov M, Baker J, Phan L, Smigielski EM, et al. dbSNP: the NCBI database of genetic variation. Nucleic Acids Res. 2001; 29(1):308-11. https://doi.org/10.1093/nar/29.1.308.

21. Li H, Handsaker B, Wysoker A, Fennell T, Ruan J, Homer N, et al. 1000 genome project data processing Subgroup. 2009. The sequence alignment/ map format and samtools. Bioinformatics. 2009;25(16):2078-9. https://doi. org/10.1093/bioinformatics/btp352.

22. Auer $\mathrm{PL}$, Johnsen JM, Johnson $A D$, Logsdon $B A$, Lange LA, Nalls MA, et al. Imputation of exome sequence variants into population-based samples and blood-cell-trait-associated loci in African Americans: NHLBI GO exome sequencing project. Am J Hum Genet. 2012;91(5):794-808. https://doi.org/1 0.1016/j.ajhg.2012.08.031

23. Wang Q, Pierce-Hoffman E, Cummings BB, Alföldi J, Francioli LC, Gauthier $\mathrm{LD}$, et al. Landscape of multi-nucleotide variants in 125,748 human exomes and 15,708 genomes. Nat Commun. 2020;11(1):1-13. https://doi.org/10.103 8/s41467-019-12438-5.

24. Karczewski KJ, Weisburd B, Thomas B, Solomonson M, Ruderfer DM, Kavanagh D, et al. The ExAC browser: displaying reference data information from over 60000 exomes. Nucleic Acids Res. 2017:45(D1):D840-D5. https:// doi.org/10.1093/nar/gkw971.

25. Ng PC, Henikoff S. SIFT: predicting amino acid changes that affect protein function. Nucleic Acids Res. 2003;31(13):3812-4. https://doi.org/10.1093/nar/ gkg509.

26. Adzhubei I, Jordan DM, Sunyaev SR. Predicting functional effect of human missense mutations using PolyPhen-2. Curr Protoc Hum Genet. 2013;76(1):7. 20. 1-7. 41. https://doi.org/10.1002/0471142905.hg0720s76.

27. Schwarz JM, Rödelsperger C, Schuelke M, Seelow D. MutationTaster evaluates disease-causing potential of sequence alterations. Nat Methods. 2010;7(8):575-6. https://doi.org/10.1038/nmeth0810-575.

28. Choi Y, Chan AP. PROVEAN web server: a tool to predict the functional effect of amino acid substitutions and indels. Bioinformatics. 2015;31(16): 2745-7. https://doi.org/10.1093/bioinformatics/btv195.
29. Untergasser A, Nijveen H, Rao X, Bisseling T, Geurts R, Leunissen JA. Primer3Plus, an enhanced web interface to Primer3. Nucleic Acids Res. 2007; 35(suppl_2):W71-W4. https://doi.org/10.1093/nar/gkm306.

30. Glaser F, Pupko T, Paz I, Bell RE, Bechor-Shental D, Martz E, et al. ConSurf: identification of functional regions in proteins by surface-mapping of phylogenetic information. Bioinformatics. 2003;19(1):163-4. https://doi.org/1 0.1093/bioinformatics/19.1.163.

31. Karolchik D, Baertsch R, Diekhans M, Furey TS, Hinrichs A, Lu Y, et al. The UCSC genome browser database. Nucleic Acids Res. 2003;31(1):51-4. https:// doi.org/10.1093/nar/gkg129.

32. Wiel L, Baakman C, Gilissen D, Veltman JA, Vriend G, Gilissen C. MetaDome: pathogenicity analysis of genetic variants through aggregation of homologous human protein domains. Hum Mutat. 2019;40(8):1030-8. https://doi.org/10.1002/humu.23798.

33. Fattahi Z, Beheshtian M, Mohseni M, Poustchi H, Sellars E, Nezhadi SH, et al. Iranome: a catalog of genomic variations in the Iranian population. Hum Mutat. 2019;40(11):1968-84. https://doi.org/10.1002/humu.23880.

34. Daneshjoo O, Garshasbi M. Novel compound heterozygote mutations in the ATP7B gene in an Iranian family with Wilson disease: a case report. J Med Case Rep. 2018;12(1):1-7. https://doi.org/10.1186/s13256-018-1608-0.

35. Desmet F-O, Hamroun D, Lalande M, Collod-Béroud G, Claustres M, Béroud C. Human Splicing Finder: an online bioinformatics tool to predict splicing signals. Nucleic Acids Res. 2009;37(9):e67-e. https://doi.org/10.1093/nar/ gkp215.

36. Spradling AC, Stern D, Beaton A, Rhem EJ, Laverty T, Mozden N, et al. The Berkeley Drosophila genome project gene disruption project: single Pelement insertions mutating 25\% of vital Drosophila genes. Genetics. 1999; 153(1):135-77.

37. Livak KJ, Schmittgen TD. Analysis of relative gene expression data using real-time quantitative PCR and the $2-\Delta \Delta C T$ method. Methods. 2001;25(4): 402-8. https://doi.org/10.1006/meth.2001.1262.

38. Hayat Nosaeid M, Mahdian R, Jamali S, Maryami F, Babashah S, Maryami F, et al. Validation and comparison of two quantitative real-time PCR assays for direct detection of DMD/BMD carriers. Clin Biochem. 2009;42(12):1291-9. https://doi.org/10.1016/j.clinbiochem.2009.04.016.

39. Soreq L, Rose J, Soreq E, Hardy J, Trabzuni D, Cookson MR, et al. Major shifts in glial regional identity are a transcriptional hallmark of human brain aging. Cell Rep. 2017;18(2):557-70. https://doi.org/10.1016/j.celrep.2016.12.011.

40. Wang Q, Ding S-L, Li Y, Royall J, Feng D, Lesnar P, et al. The Allen mouse brain common coordinate framework: a 3D reference atlas. Cell. 2020;181(4): 936-953.e20. https://doi.org/10.1016/j.cell.2020.04.007.

41. Xiong J, Ren J, Luo L, Horowitz M. Mapping histological slice sequences to the Allen mouse brain atlas without 3D reconstruction. Front Neuroinform. 2018;12:93. https://doi.org/10.3389/fninf.2018.00093.

42. Hill DP, Begley DA, Finger JH, Hayamizu TF, McCright IJ, Smith CM, et al. The mouse Gene Expression Database (GXD): updates and enhancements. Nucleic Acids Res. 2004;32(suppl_1):D568-D71. https://doi.org/10.1093/nar/ gkh069.

43. Kabzinska D, Mierzewska H, Senderek J, Kochanski A. Warburg micro syndrome type 1 associated with peripheral neuropathy and cardiomyopathy. Folia Neuropathol. 2016;3:273-81. https://doi.org/10.5114/ fn.2016.62537.

44. Hozhabri H, Talebi M, Mehrjardi MY, De Luca A, Dehghani M. Martsolf syndrome with novel mutation in the TBC1D20 gene in a family from Iran. Am J Med Genet A. 2020;182(5):957-61. https://doi.org/10.1002/a jmg.a.61543.

45. Geyer M, Wittinghofer A. GEFs, GAPs, GDIs and effectors: taking a closer (3D) look at the regulation of Ras-related GTP-binding proteins. Curr Opin Struct Biol. 1997;7(6):786-92. https://doi.org/10.1016/s0959-440x(97)80147-9.

46. Pfeffer SR. Rab GTPases: master regulators of membrane trafficking. Curr Opin Cell Biol. 1994;6(4):522-6. https://doi.org/10.1016/0955-0674(94)90071-x.

47. Ingmundson A, Delprato A, Lambright DG, Roy CR. Legionella pneumophila proteins that regulate Rab1 membrane cycling. Nature. 2007;450(7168):3659. https://doi.org/10.1038/nature06336.

48. Handley MT, Aligianis IA. RAB3GAP1, RAB3GAP2 and RAB18: disease genes in Micro and Martsolf syndromes. Biochem Soc Trans. 2012;40(6). https://doi. org/10.1042/bst20120169.

49. Sakane A, Manabe S, Ishizaki H, Tanaka-Okamoto M, Kiyokage E, Toida K, et al. Rab3 GTPase-activating protein regulates synaptic transmission and plasticity through the inactivation of Rab3. Proc Natl Acad Sci. 2006;103(26): 10029-34. https://doi.org/10.1073/pnas.0600304103. 
50. Tenawi S, Al Khudari R, Alasmar D. Novel mutation in the RAB3GAP1 gene, the first diagnosed Warburg Micro syndrome case in Syria. Oxford Med Case Rep. 2020;2020(4):omaa031. https://doi.org/10.1093/omcr/omaa031.

51. Brogna S, Wen J. Nonsense-mediated mRNA decay (NMD) mechanisms. Nat Struct Mol Biol. 2009;16(2):107-13. https://doi.org/10.1038/nsmb.1550.

52. Roest PA, Roberts RG, Sugino S, van Ommen G-JB, den Dunnen JT. Protein truncation test (PTT) for rapid detection of translation-terminating mutations. Hum Mol Genet. 1993;2(10):1719-21. https://doi.org/10.1093/ $\mathrm{hmg} / 2.10 .1719$.

53. Chang Y-F, Imam JS, Wilkinson MF. The nonsense-mediated decay RNA surveillance pathway. Annu Rev Biochem. 2007;76(1):51-74. https://doi.org/1 0.1146/annurev.biochem.76.050106.093909.

54. Asahina M, Endoh Y, Matsubayashi T, Fukuda T, Ogata T. Novel RAB3GAP1 compound heterozygous mutations in Japanese siblings with Warburg micro syndrome. Brain Dev. 2016;38(3):337-40. https://doi.org/10.1016/j.bra indev.2015.09.006.

55. Biesecker LG, Harrison SM. The ACMG/AMP reputable source criteria for the interpretation of sequence variants. Genet Med. 2018;20(12):1687-8. https:// doi.org/10.1038/gim.2018.42.

56. Gumus E. Case report of four siblings in Southeast Turkey with a novel RAB3GAP2 splice site mutation: Warburg micro syndrome or Martsolf syndrome? Ophthalmic Genet. 2018;39(3):391-5. https://doi.org/10.1080/13 816810.2018.1432065.

57. Koparir A, Karatas OF, Yilmaz SS, Suer I, Ozer B, Yuceturk B, et al. Revealing the functions of novel mutations in RAB3GAP1 in Martsolf and Warburg micro syndromes. Am J Med Genet A. 2019;179(4):579-87. https://doi.org/1 0.1002/ajmg.a.61065

\section{Publisher's Note}

Springer Nature remains neutral with regard to jurisdictional claims in published maps and institutional affiliations.

Ready to submit your research? Choose BMC and benefit from:

- fast, convenient online submission

- thorough peer review by experienced researchers in your field

- rapid publication on acceptance

- support for research data, including large and complex data types

- gold Open Access which fosters wider collaboration and increased citations

- maximum visibility for your research: over $100 \mathrm{M}$ website views per year

At BMC, research is always in progress.

Learn more biomedcentral.com/submissions 